\author{
IDENTIFICANDO A EDUCAÇÃO PERINATAL \\ OFERTADA A GESTANTES PELA EQUIPE DE EN- \\ FERMAGEM
}

\title{
IDENTIFYING THE PERINATAL EDUCATION OF- FERED TO PREGNANT WOMEN BY THE NURSING \\ TEAM
}

\author{
Willyane Paixão de Macêdo ${ }^{1}$ \\ Bruna de Souza Miranda Rio Lima \\ Lucídia de Medeiros Tavares ${ }^{3}$ \\ Claudia Maria de Souza ${ }^{4}$
}

\begin{abstract}
Resumo: Educação perinatal de e bem-estar da mãe e do bebê. tem por finalidade preparar as Pois, sabemos que no universo mulheres através de informações feminino, uma das fases mais baseadas em evidências cientí- importantes na vida de algumas ficas sobre suas opções, dando mulheres é a maternidade. Este conhecimento que lhe permitam estudo tem como objetivo geral realizar escolhas conscientes do identificar ações de educação peque favorece a melhoria na saú- rinatal realizada as gestantes pela
\end{abstract}

\footnotetext{
1 Enfermeira pelo Centro Universitário Estácio de Sá - Recife/PE

2 Enfermeira pelo Centro Universitário Estácio de Sá - Recife/PE

3 Enfermeira Especialista em Pneumologia Sanitária pelo Instituto Hélio Fraga - RJ. Mestre em Enfermagem pela UFPB. Doutora em Ciências da Educação - UNINTER

4 Fisioterapeuta e Doutora em Ciências da Educação - UNIN-

TER. Docente do UNIPE e da FTM João Pessoa/PB
}

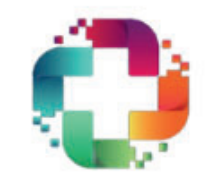


ISSN: 2763-5724

Vol. 01 - n 02 - ano 2021

Editora Acadêmica Periodicojs

equipe de enfermagem durante o pré-natal, evitando possíveis complicações. Trata-se de uma revisão integrativa da literatura, onde foram utilizados documentos já elaborados por outros autores dentre eles os artigos científicos e documentos publicados em base eletrônica, que abordem o tema educação perinatal ofertada a gestantes pela equipe de enfermagem. Os resultados apresentados sem dúvidas demonstram que a gravidez é sempre marcada por aspectos diferentes, objetivos e subjetivos, para cada mulher. Para muitas, o fato de ser mãe é algo capaz modificar seus planos e transformar suas vidas. Assim, para que a gravidez transcorra de maneira segura é necessária e imprescindível a realização do pré-natal. Conclui-se, portanto, que o enfermeiro é um profissional habilitado para acompanhar gestações. Nesse contexto, o pré-natal é um processo de acompanhamento da gestante, servindo de aprendizagem para a mulher e para a família, além disso, pode-se nesse período detectar anormalidades com a mãe e com a criança. Durante as consultas de enfermagem devem ser proporcionadas orientações de medidas favoráveis à uma abordagem apropriada das necessidades peculiares das gestantes com quem os enfermeiros interagem nas unidades de saúde durante as consultas de pré-natal.

Palavras-chave: Orientação; Gravidez; Maternidade; Enfermagem.

\begin{abstract}
Perinatal education aims to prepare women through scientific based evidence information about their options, providing knowledge that allows them
\end{abstract}


Vol. 01 - n 02 - ano 2021

Editora Acadêmica Periodicojs

to make conscious choices that favor the improvement in health and well-being of the mother and baby. Knowing that in the female universe, one of the most important phases in the life of some women is motherhood. This study aims to identify perinatal education actions performed by pregnant women by the nursing team during prenatal care, avoiding possible complications. This is an integrative literature review, using documents already prepared by other authors, including scientific articles and documents published on an electronic basis, addressing the theme of perinatal education offered to pregnant women by the nursing team. The results presented without a doubt demonstrate that pregnancy is always marked by different, objective and subjective aspects, for each woman. For many, being a mother can change their plans and transform their lives. Thus, for the pregnancy to proceed safely, prenatal care is necessary and essential. It concluded, therefore, that the nurse is a professional qualified to monitor pregnancies. In this context, prenatal care is a process of monitoring the pregnant woman, serving as a learning experience for the woman and the family. In addition, during this period, abnormalities can be detected with the mother and the child. During nursing consultations, guidelines for favorable measures should be provided for a specific approach to the specific needs of pregnant women with whom nurses interact in health units during prenatal consultations.

Keywords: Orientation. Pregnancy. Maternity. Nursing.

\section{INTRODUÇÃO}


ISSN: 2763-5724

Vol. 01 - n 02 - ano 2021

Editora Acadêmica Periodicojs

A gravidez é caracteri- ciclo de gravidez, onde as ges-

zada como um período de mudanças no corpo e emocionais que se faz necessário o acompanhamento pré-natal, com a prioridade do acolhimento à mulher, e onde é desenvolvida práticas educativas, onde as gestantes obtém orientações sobre sua gravidez e sua fisiologia, assim como respostas para seus anseios e sentimentos de medo, dúvidas, angústias.

Portanto, os profissionais de saúde da atenção básica, desempenham importante papel, no desenvolvimento de práticas educativas em saúde por estarem mais próximos da população, são capazes de reconhecer momentos críticos e intervir com seu conhecimento, que pode ser decisivo no bem estar da mulher e do seu bebê. Dentre eles, a participação do enfermeiro como membro da equipe de saúde que presta assistência direta à mulher durante o tantes demostrem satisfação nas consultas realizadas por enfermeiros através do acolhimento e à escuta que são privilegiados por estes profissionais.

Segundo Lima (2016, p. 22), durante o período do pré-natal é possível contribuir para a melhora da assistência que é prestada às gestantes. Através da mesma, é possível reduzir os índices de morbimortalidade materna e perinatal. Vale ressaltar que a gestante é o foco principal do processo de acolhimento durante sua gestação. É de suma importância relatar que o profissional enfermeiro desempenha um papel importante nesse momento, ele se torna a referência dessa mulher, sendo visto como um profissional que lhe passa confiança, estando sempre disponível para ela, já que muitas vezes a gravidez propicia momentos de 
ISSN: $2763-5724$

Vol. 01 - n 02 - ano 2021

Editora Acadêmica Periodicojs

insegurança, medo e incertezas. melhor impressão possível. A sa-

Para Marcon (2016, p. tisfação pode ser observada pela 47), o enfermeiro é um profissioreação da usuária diante do connal habilitado para acompanhar texto, do processo e do resultado gestações de baixo risco. Nesse global da experiência vivenciada. contexto, o pré-natal é um processo de acompanhamento da gestante, servindo de aprendizagem para a mulher e para a família, além disso, pode-se nesse período detectar anormalidades com a mãe e com a criança. Durante as consultas de enfermagem devem ser proporcionadas orientações de medidas favoráveis à uma abordagem apropriada das necessidades peculiares das gestantes com quem os(as) enfermeiros(as) interagem nas unidades de saúde durante as consultas de pré-natal.

Brito (2018) nos relata que muitas vezes o pré-natal passa a ser o primeiro contato da paciente com o sistema de saúde e por isso a equipe deve ter a preocupação de causar na gestante a Além disso, a opinião dos usuários acerca do atual quadro do sistema de saúde, pode também ser influenciada por diferentes expectativas, situações, antecedentes, valores, ideias e crenças. Reforçando-se, ainda, que as ações de saúde precisam estar voltadas para cobertura de toda a população alvo da área de abrangência da unidade de saúde, assegurando a continuidade no atendimento, o acompanhamento e a avaliação dessas ações sobre a saúde materna-perinatal. Assim, segundo Gonçalves (2015, p. 34) a assistência pré-natal configura-se como um momento privilegiado para se refletir, discutir e esclarecer questões que são únicas para cada mulher e seu parceiro, numa

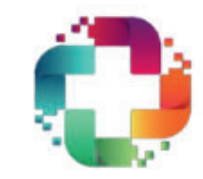


ISSN: 2763-5724

Vol. 01 - n 02 - ano 2021

Editora Acadêmica Periodicojs

abordagem individualizada, até mesmo para quem já teve outros filhos anteriormente.

Sabemos que a gestação desenvolve inúmeras e diferentes ações dentre elas a promoção da saúde da mulher, a prevenção de doenças e o tratamento de diversas patologias que podem vir a surgir no decorrer da gravidez, por meio do acolhimento e orientação de consultas médicas e dos enfermeiros.

O acompanhamento do pré-natal é um conjunto de exames realizados durante a gestação que possibilitam que a mulher faça um acompanhamento detalhado da sua saúde e do bebê. Segundo o Ministério da Saúde (2014/2015), cada vez mais o número de atendimento às gestantes, na atenção da rede básica de saúde, vem aumentando, acredita-se que o ocorrido deste fato, é devido à qualidade da assistên- cia prestada pelos serviços e pelos profissionais de saúde, quanto a transmissão em educação em saúde.

Esse cuidado ajuda a prevenir diversas doenças e complicações que podem trazer inclusive o parto prematuro e o aborto. Por isso a realização do pré-natal representa papel fundamental na prevenção e detecção precoce de patologias tanto maternas como fetais, permitindo um desenvolvimento saudável do bebê e reduzindo os riscos da gestante. Informações sobre as diferentes vivências devem ser trocadas entre as mulheres e os profissionais de saúde. Essa possibilidade de experiências e conhecimentos é considerada a melhor forma de promover a compreensão do processo de gestação.

Partindo desses pressupostos surgiu então a seguinte pergunta norteadora do estudo:

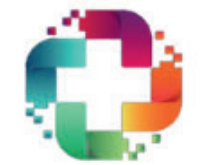


Como os enfermeiros realizam um atendimento assegurado na educação perinatal as gestantes?

Justifica-se a importância da participação do enfermeiro e sua contribuição evidenciando sentimentos, anseios e preocupações das gestantes, que muitas vezes estão fragilizadas, o que é relevante tanto para a comunidade acadêmica, para os profissionais de saúde quanto para o serviço de saúde, pois se caracteriza como a porta de entrada para investigar carências, necessidades e sentimentos das gestantes.

O estudo tem por objetivo identificar ações de educação realizada para gestantes pela equipe de enfermagem durante o pré-natal, evitando possíveis complicações. Dessa forma o pré-natal deve ser organizado para atender às reais necessidades das gestantes, por meio da utilização de conhecimentos técnico-científicos, recursos adequados e disponíveis para cada caso. Por isso a pesquisa é fundamentada na demonstração da participação dos enfermeiros em assegurar o pré-natal as gestantes inseridas nos serviços de saúde.

\section{METODOLOGIA}

A presente pesquisa trata de uma revisão integrativa da literatura sobre a educação perinatal ofertada a gestantes pela equipe de enfermagem. Esta modalidade de pesquisa permite a análise de pesquisas e síntese dos conceitos de forma ampla, tendo em vista a necessidade do conhecimento científico para elaboração e desenvolvimento do artigo. Algumas etapas foram seguidas para elaboração da revisão integrativa, sendo elas: Elaboração da pergunta/problemática; coleta bibliográfica, classificação dos 
ISSN: $2763-5724$

Vol. 01 - n 02 - ano 2021

Editora Acadêmica Periodicojs

dados, análise e discussão dos estudos incluídos, e resultados (CROSSETTI, 2014).

A revisão integrativa da literatura é um método que consiste em analisar de forma crítica, estudos anteriores sobre a temática selecionando-os de acordo com as fases organizativas que permitem analisar e avaliar os dados coletados. Devido à alta demanda de informações na área da saúde, se faz necessário a triagem dos artigos subsidiados em evidências comprovadas para pesquisas científicas. A partir desta premissa, a revisão integrativa proporciona uma organização dos resultados mais relevantes das pesquisas (CROSSETTI, 2014).

Através da busca em periódicos nacionais, na base de dados (SCIELO), PUBMED em Ciências de Saúde (LILACS), os artigos selecionados foram entre os anos de 2014 a 2019. Após o refinamento, oito artigos contemplaram o objetivo proposto e os critérios de inclusão, os dados foram organizados quanto a identificação do artigo, instituição do estudo, tipo de publicação, características e rigor metodológico, seguindo as etapas da revisão integrativa: identificação do tema, elaboração da pergunta norteadora, busca ou amostragem na literatura, coleta de dados, análise crítica dos estudos incluídos, discussão dos resultados e apresentação da revisão integrativa.

\section{RESULTADOS E DISCUS- SÃO}

Após a identificação dos oito artigos que atenderam aos critérios de inclusão previamente estabelecidos, foram conceitualizados o pré-natal e o acompanhamento perinatal ofertada

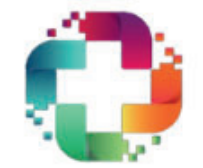


Vol. 01 - n 02 - ano 2021

Editora Acadêmica Periodicojs

as gestantes pelos enfermeiros em determinadas comunidades. Após as leituras, foi possível detectar os diversos enfoques na perspectiva da temática produzidos no campo da educação perinatal, ou seja, a participação dos enfermeiros nas consultas das gestantes para o acompanhamento do seu pré-natal.

Conforme Oliveira (2018), o enfermeiro precisa realizar consultas de pré-natal qualificadas. A consulta de pré-natal qualificada deve seguir um roteiro básico com atendimento aos aspectos sociais, epidemiológicos, antecedentes pessoais, ginecológico, sexuais e obstétricos e dados sobre a gestão atual. Pois, o enfermeiro pode solicitar exame e encaminhar para outros profissionais de saúde para que o acompanhamento seja processado de forma integral.

$\mathrm{O}$ autor ainda relata que os registros das consultas devem sempre estar na caderneta da gestante, é uma ação importante para assistência ao pré-natal nos serviços de saúde, para que o cuidado seja contínuo, com completo histórico da gestação e assistência do parto.

Segue abaixo no quadro as características dos artigos da amostra quanto a autoria, titulação, periódico, ano de publicação e tipo de estudo, bem como seus objetivos e resultados 
ISSN: 2763-5724

Vol. 01 - n 02 - ano 2021

Editora Acadêmica Periodicojs

\begin{tabular}{|c|c|c|c|c|c|c|}
\hline $\begin{array}{l}\text { Bawe } \\
\text { Ano }\end{array}$ & Autar & Titulo & Obstivo & Máado & Reavitado & NE* \\
\hline 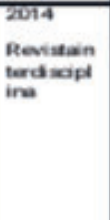 & $\begin{array}{l}\text { CACEDDAL } \\
\text { M. ELIAS. } \\
\text { C. } \\
\text { MIRANDAR, } \\
\text { A. } \\
\text { FETOSA } \\
\text { V.C } \\
\text { RIBEIRO. I }\end{array}$ & 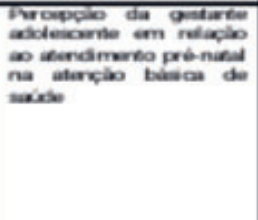 & 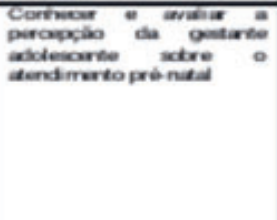 & 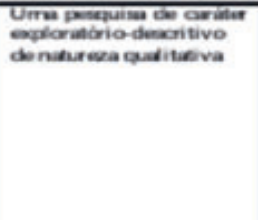 & 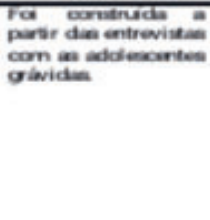 & 5 \\
\hline $\begin{array}{l}\text { 2015 } \\
\text { Pevisain } \\
\text { terdacipl } \\
\text { ine }\end{array}$ & $\begin{array}{l}\text { PODWDOUES } \\
\text { SA. } \\
\text { SILVA.K. } \\
\text { RIBEIRO } \\
\text { T.C.O }\end{array}$ & 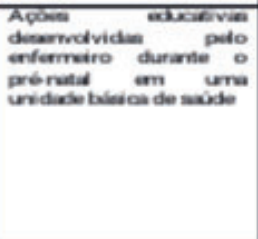 & 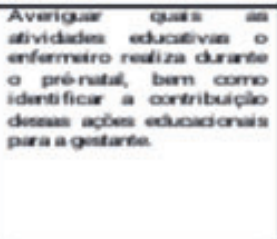 & 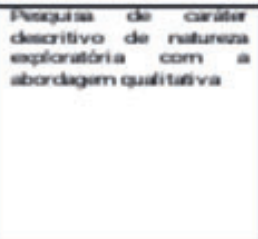 & 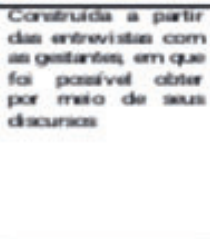 & $\frac{2}{2}$ \\
\hline $\begin{array}{l}\text { 2015 } \\
\text { Litacs } \\
\text { Rew } \\
\text { Enferm } \\
\text { Ursm }\end{array}$ & $\begin{array}{l}\text { CARVALHO } \\
\text { MDAB } \\
\text { ORTICAES } \\
\text { F. } \\
\text { OELLOSO } \\
\text { SM. }\end{array}$ & 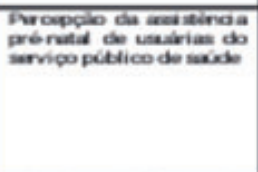 & 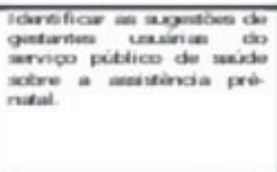 & 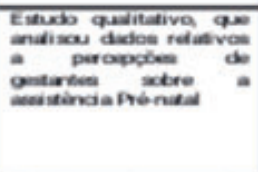 & 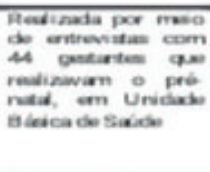 & 0 \\
\hline 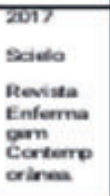 & $\begin{array}{l}\text { POCFKCA } \\
\text { ANORADE } \\
\text { C.S }\end{array}$ & 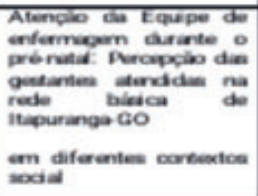 & 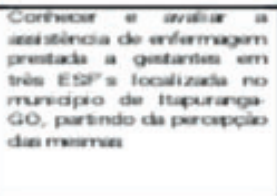 & 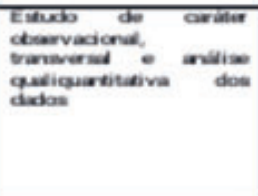 & 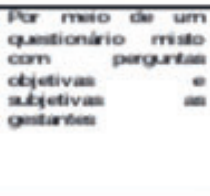 & 14 \\
\hline $\begin{array}{l}2017 \\
\text { Litaca } \\
\text { Revist } \\
\text { Saido }\end{array}$ & $\begin{array}{l}\text { CUEDESC } \\
\text { SOUZA,YM } \\
\text { EDEIROS L. } \\
\text { SILVA.A } \\
\text { ARAUDO N } \\
\text { BELISANA } \\
\text { P }\end{array}$ & 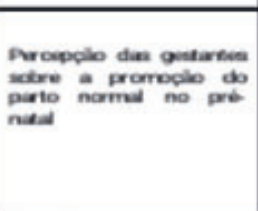 & 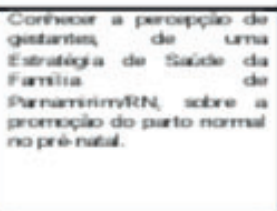 & 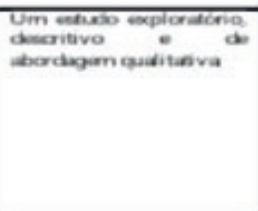 & 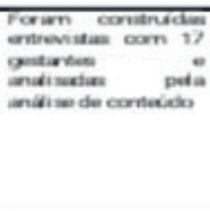 & 6 \\
\hline $\begin{array}{l}\text { 2010 } \\
\text { Ren inse } \\
\text { Cierd * } \\
\text { Ext }\end{array}$ & $\begin{array}{l}\text { OLIVEIRAB } \\
\text { CD } \\
\text { BaITOSS } \\
\text { ClOTrOAC }\end{array}$ & 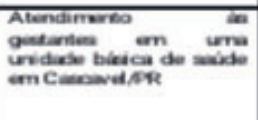 & 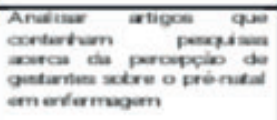 & 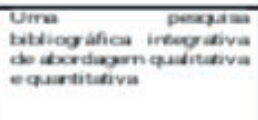 & 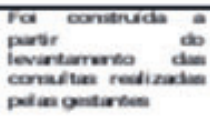 & 5 \\
\hline $\begin{array}{l}\text { 2010 } \\
\text { Liaca } \\
\text { Revis }\end{array}$ & 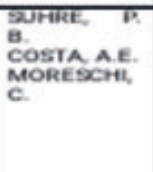 & 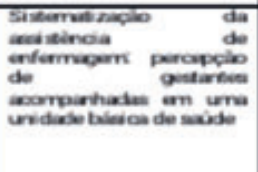 & 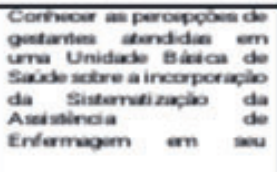 & 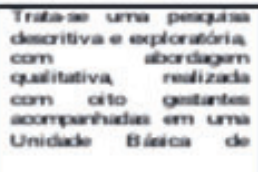 & 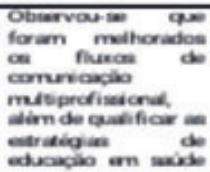 & $\mathbf{3}$ \\
\hline
\end{tabular}

Fonte: Dados literários da pesquisa, 2020. Recife/PE, Brasil.

$\mathrm{Na}$ apresentação dos relatava em relação ao tema.

resultados foi realizada uma

comparação com os objetivos da

CATEGORIZAÇÃO TEMÁ-

pesquisa de forma descritiva bus-

TICA DOS ESTUDOS/SUB-

cando conhecer o que cada artigo

\author{
CATEGORIAS
}

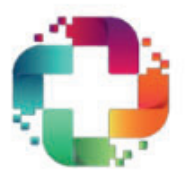

HEALTH \& SOCIETY 
Vol. 01 - n 02 - ano 2021

Editora Acadêmica Periodicojs

é um acompanhamento durante

A pesquisa busca co- o ciclo gestacional, cujo obje-

nhecer através de uma revisão integrativa da literatura sobre a educação perinatal ofertada a gestantes pela equipe de enfermagem. Após a leitura dos artigos, os dados analisados foram sintetizados e categorizados em confronto com os objetivos desta pesquisa e subdivididos nos seguintes temas: Assistência Pré-Natal realizado pelos enfermeiros nas gestantes; Contribuições das ações educativas para a gestante; Avaliação da gestante acerca do atendimento pré-natal.

ASSISTÊNCIA PRÉ-NATAL

Em todos os artigos ficou claro a importância da assistência a gestante durante o pré-natal, onde apresenta-se a seguir os relatos. Para Lacerda et al. (2014) a assistência pré-natal tivo primário é o acolhimento da mulher desde o início de sua gravidez, garantindo que ao termino desta, ocorra o nascimento de uma criança saudável, assim como a garantia do bem estar materno e neonatal.

Durante a gestação a adolescente passa por múltiplas alterações e manifestações biológicas, que ocorrem paralelas às psicológicas. Estas podem se apresentar na forma de nervosismo, medo, tensão ou insegurança, por exemplo. Para a adaptação a essa nova etapa de vida é relevante que a adolescente grávida esteja inserida num contesto favorável no qual seja proporcionado o apoio familiar e cuidados médicos e de enfermagem. (RODRIGUES et al., 2015)

Nesse âmbito, Carvalho et al. (2015) aponta que as ações 
ISSN: 2763-5724

Vol. 01 - n 02 - ano 2021

Editora Acadêmica Periodicojs

indicativas para uma boa assistência pré-natal são os esclarecimentos do profissional sobre educação e promoção da saúde que incluem as orientações entendidas como um conjunto de conhecimentos, práticas e atitudes que visam à promoção do parto espontâneo e também a prevenção da morbidade e mortalidade maternas.

Andrade et al. (2017) afirma que nesse momento, a gestante deverá receber as orientações necessárias referentes ao acompanhamento pré-natal. Desse modo, ao entrar em contato com os profissionais da área, seja no centro de saúde ou na comunidade, ela deve buscar informações quanto à compreensão da fase gestacional, no que deve ser redobrada a atenção quando ela for adolescente.

O pré-natal é o acompanhamento voltado as gestantes.
Conceituado como um conjunto de ações que antecedem ao parto, tendo por finalidade atender as necessidades da mulher, promovendo a qualidade de vida e prevenido intercorrências (GUEDES et al., 2017).

Oliveira et al. (2018) o pré-natal tem contribuído de forma notável para a saúde pública, diminuindo o risco de complicações tanto no pré-natal quanto no pós-parto, através de consultas periódicas com enfermeiros. As consultas de enfermagem são baseadas nos conceitos de prevenção, promoção e vigilância da saúde, que visa garantir o bem-estar e melhor qualidade de vida para as gestantes.

Para Moreschi et al. (2018), as gestantes estão buscando o pré-natal devido à qualidade da assistência prestada pelo serviço e profissionais de saúde, uma vez que elas estão cada dia 
ISSN: 2763-5724

Vol. 01 - n 02 - ano 2021

Editora Acadêmica Periodicojs

mais consciente da importância deste atendimento para a diminuição dos elevados índices de mortalidade materno-fetal.

Para a realização de um pré-natal de qualidade é necessário um conjunto de recursos que compreende: recursos humanos, área física adequada, equipamentos e instrumentos mínimos, apoio laboratorial, material para registro, processamento, analise de dados e medicamentos. (SOUZA et al., 2019)

\section{CONTRIBUIÇÕES DAS} AÇÕES EDUCATIVAS PARA A GESTANTE

Os artigos apresentados mostram relatos onde os autores apresentam ideias semelhantes.

Lacerda (2014) e Rodrigues (2015) relatam que ao realizar as atividades de orientação/informação, o enfermeiro deve ter a compreensão de que está contribuindo profundamente para a educação em saúde, valendo avançar para o alcance da educação como meta maior. A adoção de práticas benéficas à saúde, bem como a mudança para comportamentos favoráveis à saúde é o resultado esperado de um bom programa de educação em saúde.

As ações educativas em saúde são processos que objetivam capacitar indivíduos e/ ou grupos, de modo que possam assumir a responsabilidade de ajudar na melhoria das condições de saúde da população. As autoras acrescentam, ainda, que os profissionais e a população devem compreender que as ações oferecidas pelos serviços de saúde, assim como o esforço da própria população, mediante conhecimentos, motivação, reflexão e adoção de novas práticas de

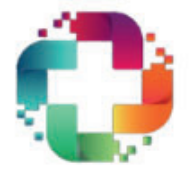


ISSN: 2763-5724

Vol. 01 - n 02 - ano 2021

Editora Acadêmica Periodicojs

saúde, é que irão garantir a saúde da comunidade (CARVALHO, 2015; ANDRADE, 2017; GUEDES, 2017).

Para Oliveira (2018), Moreschi (2018) e Souza (2019) nos questionam que a prática de comunicação e informação em saúde pode ser desenvolvida por meio de uma proposta de sistematização da assistência de enfermagem, de maneira que sejam identificadas as principais necessidades de informação do cliente, da família e da comunidade, e ao serem atendidas, possam os efeitos ser monitorados com relação ao impacto sobre o comportamento e a vida das pessoas.

AVALIAÇÃO DA GESTANTE ACERCA DO ATENDIMENTO PRÉ-NATAL

Para Lacerda (2014) e Rodrigues (2015) as gestan- tes tornaram evidente por meio de suas exposições, a satisfação com o atendimento recebido pelo enfermeiro, assim manifestando a importância de que para haver processo gravídico satisfatório se faz necessária assistência de qualidade por parte da equipe

O pré-natal é indicado para todas as mulheres grávidas, pois é acompanhamento fundamental para alcançar um parto sem maiores complicações. Assim que a mulher perceber que está grávida, necessita procurar os serviços de saúde, para realização da mesma comunidade (CARVALHO, 2015; ANDRADE, 2017).

A gestante deve iniciar o pré-natal no primeiro trimestre de gravidez, devendo ter no mínimo de 06 (seis) consultas, sendo 01 (uma) no primeiro trimestre, 02 (duas) no segundo trimestre e 03 (três) no terceiro 
trimestre. As consultas poderão ser realizadas tanto na Unidade de Saúde quanto durante a visita domiciliar, e o intervalo entre as consultas deve ser de 04 (quatro) semanas (GUEDES, 2017; OLIVEIRA, 2018).

Moreschi (2018) e Souza (2019) nos afirmam que o diagnóstico da gravidez e as consultas de pré-natal podem ser feitas tanto pelo médico quanto pelo enfermeiro da Unidade Básica. Nestas, o profissional deverá fornecer a gestante, o seu cartão, com a identificação preenchida e orientação sobre o mesmo; o calendário de vacinas e suas orientações; a solicitação de exames de rotina; as orientações sobre atividades educativas. Estas ações visam identificar e prevenir intercorrências que possam trazer agravos à gestante ou ao bebê.

\section{CONCLUSÃO}

Conclui-se para que a gestação transcorra de maneira saudável, com ganhos para mães e bebês, é necessário que a gestante mantenha a regularidade preconizada pelo Ministério da Saúde no que diz respeito ao número de consultas ao longo desse período. Portanto, o acompanhamento do pré-natal pelos profissionais da saúde em especial o enfermeiro aqui citado é de sua importância a gestante. Pois, ela tem a oportunidade de se conhecer como mulher, de estar inteirada no que se refere a sua saúde e a saúde do seu filho.

Tendo em vista o levantamento e leitura dos artigos pesquisados, observou-se que existe opiniões com relatos onde as contradições são as mesmas como em relação aos dados pessoais e a satisfação com a equipe de 
ISSN: 2763-5724

Vol. 01 - n 02 - ano 2021

Editora Acadêmica Periodicojs

enfermagem. Podemos perceber como pontos principais que, no geral, o atendimento da equipe de enfermagem gera satisfação e bem-estar para as pacientes, necessitando-se de aprimoramento em alguns pontos, como mais e melhores comunicações e orientações individuais e em grupo com acolhimento humanizado e redução no tempo de espera para serem atendidas e facilidades no encaminhamento a outros serviços de saúde.

Os resultados deste estudo possibilitaram identificar as contribuições das ações educativas às gestantes, descrevendo quais atividades de comunicação/informação em saúde que o enfermeiro implementa no transcorrer da assistência pré-natal e analisar a avaliação da gestante frente ao pré-natal. Assim, a identificação dos pontos de vista e do conhecimento desse grupo sobre o pré-natal trouxe um diagnóstico valioso, possibilitando o encontro das necessidades e dos aspectos que precisam receber durante este período, principalmente no que se refere ao conhecimento e informação.

$$
\text { É durante o pré-natal }
$$
que ela esclarece qualquer dúvida, que diminui seus medos e suas angústias e a ansiedade de saber o processo natural de estar grávida. Ser acolhida nesse momento, se sentir aconchegada, manter uma relação de confiança e estabelecer vínculo com o profissional que está participando desse momento é essencial para que esse momento dê certo. $\mathrm{O}$ acolhimento é exatamente isso, ao mesmo tempo que o atendimento deve ser resolutivo, ele deve ser acolhedor, deve trazer bem-estar a essa mulher, deve vê-la como um todo, como ser humano e como gestante.

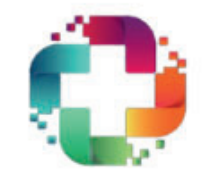


ISSN: 2763-5724

Vol. 01 - n 02 - ano 2021

Editora Acadêmica Periodicojs

Espera-se que os profissionais de saúde, em particular o enfermeiro, a desenvolver trabalhos educativos diferenciados focados nesta população específica, visando à melhoria crescente da qualidade da assistência, transformando as ações em práticas concisas, eficazes e eficientes, capazes de contribuir com a promoção da saúde materna e infantil.

É imprescindível que as gestantes saibam a importância do papel do enfermeiro no transcorrer do acompanhamento nessa fase de sua vida. Só assim, elas compreendem o acompanhamento, como uma ferramenta fundamental para reduzir o sentimento de medo, angústia, ansiedade e preocupação, além de sanar dúvidas, além de trazer informações sobre o desenvolvimento da gestação. O profissional enfermeiro desempenha um papel singular nesse momento, ele se torna a referência dessa mulher, sendo visto como um profissional que lhe passa confiança, estando sempre disponível para ela, já que muitas vezes a gravidez propicia momentos de insegurança, medo e incertezas. Além dessa importante questão que é o acolhimento, as depoentes apontaram algumas limitações estruturais na rede de saúde.

$$
\text { É importante realizar }
$$
estudos sobre as estratégias de promoção da saúde adotadas pelos enfermeiros, para que possam atender às necessidades das gestantes e proporcionar-lhes conhecimentos sobre a importância e a necessidade do pré-natal, e sobre o quanto isso influencia a saúde delas. Sabemos que sua participação neste período é de suma importância, pois sem ele, as consultas se restringem a um modelo biomédico de queixas e

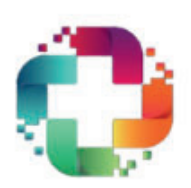


ISSN: 2763-5724

Vol. 01 - n 02 - ano 2021

Editora Acadêmica Periodicojs

solicitações de exames.

Por fim, o papel do enfermeiro é de grande relevância no que diz respeito às ações voltadas ao pré-natal, além da realização de seus procedimentos técnicos, uma das suas principais atividades é estabelecimento de relação de apoio, compreensão e discussão de aspectos fundamentais à saúde da mulher, criando assim uma relação de confiança.

\section{REFERÊNCIAS}

ANDRADE, Ana Cláudia. Atenção da Equipe de enfermagem durante o pré-natal: Percepção das gestantes atendidas na rede básica de Itapuranga-GO em diferentes contextos social. Scielo, Revista Enfermagem Contemporânea, 2017.

BRASIL, Ministério da Saúde. Atenção ao pré-natal de baixo risco: Caderno de Atenção Bási-

ca. Brasília: Ministério da Saúde, 2015.

BRASIL. Ministério da Saúde. Secretaria de Atenção à Saúde: Departamento de Ações Programáticas Estratégicas. Manual dos comitês de mortalidade materna. 3a ed. Brasília (DF): Ministério da Saúde (BR), 2014.

BRITO, S. A. C. Percepção das gestantes sobre o pré-natal. Ver. Inic. Cient. Ext., 2018.

CARVALHO, ELISANGELA, SANDRA. Percepção da assistência pré-natal de usuárias do serviço público de saúde. Lilacs. Rev. Enferm. UFSM, 2015.

CROSSETTI, M. G. O. Revisão integrativa de pesquisa na enfermagem: o rigor científico que lhe é exigido. Revista gaúcha de en-

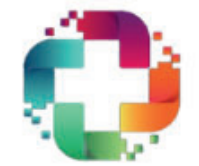


fermagem, v. 33, n. 2, 2014.

GONÇALVES, C. V. Qualidade e equidade na assistência à gestante: Saúde Pública. Rio de Janeiro, v. 25, n.11, 2015.

GUEDES, C. D. F. SOUZA, T. K. MEDEIROS, L. ; SILVA, D.A ARAÚJO N. BELISANA P. Percepção das gestantes sobre a promoção do parto normal no pré-natal. Lilacs. Revis. Scielo, 2017.

LACERDA, CONCEIÇÃO, RAFAELLA，ELIZABETH，VERBÊNIA, ÍTALO. Percepção da gestante adolescente em relação ao atendimento pré-natal na atenção básica de saúde. Revista Interdisciplinar, 2014.

LIMA, M. S. Apoio no Pré-Natal dos Enfermeiros. Rev. Esc. Enferm. USP. Fortaleza, v. 42, n. 01,
2016.

MARCON, S. S. "Flashes" de como as gestantes percebem a assistência pré-natal em um Hospital Universitário. 2016.

MORESCHI, P.B, COSTA, A. E. Sistematização da assistência de enfermagem: percepção de gestantes acompanhadas em uma unidade básica de saúde. Lilacs. Revista, 2018.

OLIVEIRA， SANDRA， ANI CÁTIA. Atendimento às gestantes em uma unidade básica de saúde em Cascavel/PR. Ver. Inic. Cient. e Ext., 2018.

RODRIGUES, SARAH, KAMILA, THAÍS. Ações educativas desenvolvidas pelo enfermeiro durante o pré-natal em uma unidade básica de saúde. Revista Interdisciplinar, 2015. 
ISSN: 2763-5724

Vol. 01 - n 02 - ano 2021

Editora Acadêmica Periodicojs

SOUZA, Z. N. R; ROSA, M.

C.; BASTIANI, J. A. N. Maternidade: percepções de gestantes

primíparas usuárias do Serviço

Básico de Saúde. Scielo, Lilacs.

Enfermagem/Nursing, 2019. 